\title{
Relationship between acetaldehyde concentration in mouth air and tongue coating volume
}

\author{
Aya YOKOI', Takayuki MARUYAMA ${ }^{1,2}$, Reiko YAMANAKA ${ }^{3}$, Daisuke EKUNI' ${ }^{1}$, Takaaki TOMOFUJI 1,4, Haruhiko \\ KASHIWAZAKI ${ }^{5}$, Yutaka YAMAZAKI ${ }^{5}$, Manabu MORITA ${ }^{1}$ \\ 1- Department of Preventive Dentistry, Okayama University Graduate School of Medicine, Dentistry and Pharmaceutical Sciences, Okayama, Japan.
2- Center for Innovative Clinical Medicine, Okayama University Hospital, Okayama, Japan.
3- Division of Hospital Dentistry, Central Clinical Department, Okayama University Hospital, Okayama, Japan.
4- Advanced Research Center for Oral and Craniofacial Sciences, Okayama University Dental School, Okayama, Japan.
5- Gerodontology, Department of Oral Health Science, Graduate School of Dental Medicine, Hokkaido University, Sapporo, Japan.
}

Corresponding address: Daisuke Ekuni - Department of Preventive Dentistry, Okayama University Graduate School of Medicine, Dentistry and Pharmaceutical Sciences - 2-5-1 Shikata-cho - Kita-ku - Okayama - 700-8558 - Japan - Phone: +81 86235 6712; Fax: +81 862356714 - e-mail: dekuni7@md.okayama-u.ac.jp

Submitted: June 3, 2014 - Modification: October 28, 2014 - Accepted: October 30, 2014

\section{ABSTRACT}

\begin{abstract}
bjective: Acetaldehyde is the first metabolite of ethanol and is produced in the epithelium by mucosal ALDH, while higher levels are derived from microbial oxidation of ethanol by oral microflora such as Candida species. However, it is uncertain whether acetaldehyde concentration in human breath is related to oral condition or local production of acetaldehyde by oral microflora. The aim of this pilot study was to investigate the relationship between physiological acetaldehyde concentration and oral condition in healthy volunteers. Material and Methods: Sixty-five volunteers (51 males and 14 females, aged from 20 to 87 years old) participated in the present study. Acetaldehyde concentration in mouth air was measured using a portable monitor. Oral examination, detection of oral Candida species and assessment of alcohol sensitivity were performed. Results: Acetaldehyde concentration [median $(25 \%, 75 \%)$ ] in mouth air was $170.7(73.5,306.3)$ $\mathrm{ppb}$. Acetaldehyde concentration in participants with a tongue coating status score of 3 was significantly higher than in those with a score of $1(p<0.017)$. After removing tongue coating, acetaldehyde concentration decreased significantly $(p<0.05)$. Acetaldehyde concentration was not correlated with other clinical parameters, presence of Candida species, smoking status or alcohol sensitivity. Conclusion: Physiological acetaldehyde concentration in mouth air was associated with tongue coating volume.
\end{abstract}

Keywords: Acetaldehyde. Tongue. Bacteria. Cross-sectional studies.

\section{INTRODUCTION}

Although metabolism of alcohol by aldehyde dehydrogenase $(A L D H)$ principally occurs in the liver ${ }^{4}$, alcohol metabolism is also known to be carried out in other areas of the body. For example, acetaldehyde is produced in the epithelium by mucosal ALDH ${ }^{2}$, while higher levels are derived from microbial oxidation of ethanol by oral microflora such as Candida species ${ }^{9,10,12,17,23}$. Thus, the effects of acetaldehyde on the oral cavity can be local, and oral hygiene may be linked to local production of acetaldehyde by oral microflora.

Acetaldehyde is known to be carcinogenic and to cause mutations in DNA ${ }^{25}$. Long-term exposure to acetaldehyde, even at physiological concentrations, may affect cell activity and cause mutations in DNA. For example, physiological concentrations of acetaldehyde (220 ppb) influence cell proliferation in rabbit aortic myocytes after long-term exposure ${ }^{27}$. Therefore, physiological concentrations of acetaldehyde in oral cavity might affect cell activity in both humans and animal models.

Only one study has reported data on physiological concentrations of acetaldehyde in human breath (0.4-1.6 ppb) in a small number of subjects $(n=20)^{5}$. However, the relationship between physiological concentrations of acetaldehyde in human breath and oral condition is uncertain. Thus, 
we hypothesized that physiological concentrations of acetaldehyde in human breath are related to oral condition or local production of acetaldehyde by oral microflora. The aim of this study was to investigate the physiological concentrations of acetaldehyde in human mouth air and the relationship between these concentrations and oral condition.

\section{MATERIAL AND METHODS}

\section{Participants}

At the Dental Clinic of Okayama University Hospital, sixty-five volunteers (51 males and 14 females, aged from 20 to 87 years old; mean age $44.0 \pm 22.7$ years) without respiratory, digestive system, otorhinolaryngologic or liver disease and not undergoing any antibiotic or other antimicrobial therapy participated in the present study, when the dentists asked the patients to participate the research. The recruitment period was from October 2013 to August 2014. The study was approved by the Ethics Committee of Okayama University Graduate School of Medicine, Dentistry and Pharmaceutical Sciences (No. 1461, August 28,2012 ). Written consent was obtained from all participants.

\section{Measurement of acetaldehyde}

We used the Sensor Gas Chromatograph SGEA-P2 (FIS Inc., Itami, Japan). The system consists of pump, filter, flow control, column, detector (semiconductor gas sensor) and sample injection area (manual injection with a syringe). As a high-sensitivity semiconductor gas sensor is used as a detector, ppb level measurement is possible. Using a syringe, injection of sample gas $(5 \mathrm{~mL})$ starts the measurement automatically. Measurement was completed in 8 minutes. The monitor uses ambient air as a carrier gas, and a high-pressure gas cylinder is not necessary. To assess the reproducibility of the portable monitor, 100-10,000 ppb acetaldehyde was used for calibration. Measurement was performed in duplicate. Both intra- and inter-assay coefficients of variation were below $5 \%$.

Participants were advised to abstain from food or drink and to refrain from their standard oral hygiene practice on the morning of the day of measurements. Participants were also instructed to refrain from eating strong smelling foods for at least $48 \mathrm{~h}$, from using strong perfumes for $24 \mathrm{~h}$, from smoking for $24 \mathrm{~h}$ and from drinking alcohol for $12 \mathrm{~h}$ prior to measurements. Actual measurements were conducted in the morning, between 8 and 9 am. Participants kept their mouths closed for 3 min prior to measurement of mouth air with a syringe $^{19}$ (Figure 1 ). During collection, participants breathed through their nose. As acetaldehyde is highly volatile, we avoided air contamination in the oral cavity as much as possible. Sample gas in the syringe was then injected immediately and measured.

We also investigated acetaldehyde concentration changes after tongue coating removal in 6 participants ( 5 males and 1 female, aged from 27 to 65 years old; mean age, $39.8 \pm 18.4$ years) with a tongue coating status score of 3 . In addition, the diurnal variation [morning (between 8:00 and 9:00), noon (between 12:00 and 1:00) and evening (between 17:00 and 18:00)] in another 6 participants ( 5 males and 1 female, aged from 27 to 41 years old; mean age, $30.2 \pm 5.4$ years) was examined.

\section{Oral examination}

Probing pocket depth (PPD) and clinical attachment level (CAL) were determined at six sites (mesiobuccal, midbuccal, distobuccal, mesiolingual, midlingual and distolingual) on all teeth using a color-coded probe (Hu-Friedy, Chicago, IL, USA). Sites that bled upon gentle probing ( $25 \mathrm{~g}$ probing force) were recorded, and the proportion of sites with bleeding on probing (BOP) was measured in each participant. The plaque control record (PCR) was measured using erythrosine staining, and was recorded with respect to their relative location to the gingival margin at four sites (mesial, distal, buccal and lingual) around each tooth ${ }^{22}$. Tongue coating status was assessed according to distribution area as follows: score 0 : none visible; 1 : less than one

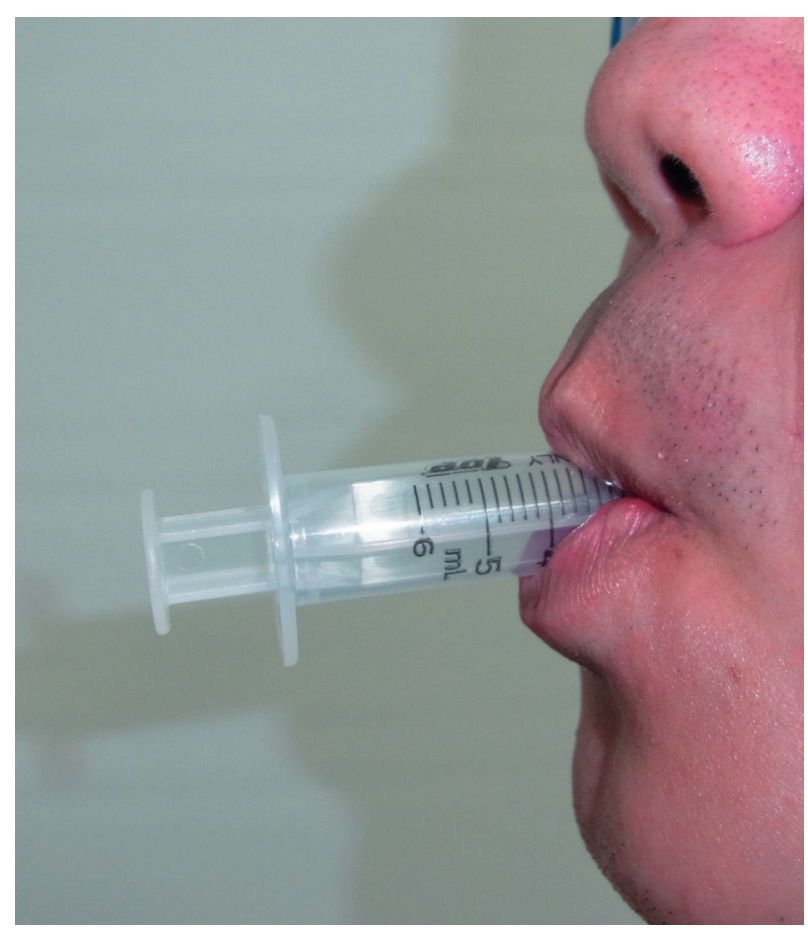

Figure 1- Collection of acetaldehyde. Participants kept their mouths closed for $3 \mathrm{~min}$. The syringe was tightly held between lips to avoid contamination of the oral cavity with outside air 
third of the tongue dorsum surface covered; 2 : less than two thirds; 3 : more than two thirds ${ }^{18}$. All clinical procedures were performed by four trained and calibrated dentists (A. Y., T. M., T. T. and D. E.). Intra- and inter-examiner agreement for the oral examination (tongue coating status, PPD and $\mathrm{CAL}$ ) was good, as evaluated by kappa statistics of more than 0.8 .

\section{Detection of Candida species}

We used a CHROMagar Candida medium (Kanto Chemical Corp., Inc., Tokyo, Japan) ( $\mathrm{pH} \mathrm{6.1)} \mathrm{to}$ detect Candida albicans (C. albicans), Candida tropicalis (C. tropicalis) and Candida krusei (C. $\left.k_{\text {rusei }}\right)^{21}$. The medium comprised (per liter) peptone $(10 \mathrm{~g})$, glucose $(20 \mathrm{~g})$, agar $(15 \mathrm{~g})$, chloramphenicol
$(0.5 \mathrm{~g})$ and Chromogenic ix ( $2 \mathrm{~g})$, and was prepared in accordance with the manufacturer's instructions. All samples wiped from the surface of buccal mucosa and tongue dorsum using a sterilized dental mirror were plated on the medium for 48 hours at $37^{\circ} \mathrm{C}$. Production of color and morphology, as described by the manufacturer, were recorded and photographs were recorded; i.e, green colonies of C. albicans, steel blue colonies of $C$. tropicalis and rose colored colonies of C. krusei ${ }^{21}$.

\section{Assessment of alcohol sensitivity}

We used the ethanol patch test (ASK Human Care Inc., Tokyo, Japan) to assess participant genotypes ${ }^{28}$. Briefly, a patch plaster fixed on adhesive tape was attached to the inner surface

Table 1- Characteristics of participants $(n=65)$

\begin{tabular}{|c|c|c|c|c|}
\hline Variable & & Male & Female & Total \\
\hline Acetaldehyde concentration (ppb) & & $175.1(87.3,322.6)^{*}$ & $99.2(55.4,236.2)$ & $170.7(73.5,306.3)$ \\
\hline Number of teeth present & & $28(26,30) \dagger$ & $24.5(22.3,27.5)$ & $28(25,30)$ \\
\hline Mean probing pocket depth (mm) & & $2.2(2.0,2.3) \dagger$ & $2.0(1.7,2.1)$ & $2.2(1.9,2.3)$ \\
\hline Mean clinical attachment level (mm) & & $2.3(2.1,2.5)$ & $2.1(1.9,2.3)$ & $2.3(2.0,2.5)$ \\
\hline Bleeding on probing (\%) & & $8.6(3.9,15.8)$ & $7.1(2.9,18.4)$ & $8.3(3.6,15.9)$ \\
\hline Plaque control record (\%) & & $43.3(23.7,60.0)$ & $38.5(18.4)$ & $39.8(21.4,60.7)$ \\
\hline \multirow[t]{4}{*}{ Tongue coating status } & 0 & $7(13.7) \ddagger$ & $1(7.1)$ & $8(12.3)$ \\
\hline & 1 & $8(15.7)$ & $2(14.3)$ & $10(15.4)$ \\
\hline & 2 & $12(23.5)$ & $6(42.9)$ & $18(27.7)$ \\
\hline & 3 & $24(47.1)$ & $5(35.7)$ & $29(44.6)$ \\
\hline Candida species & + & $13(25.5) \dagger$ & $8(57.1)$ & $21(32.3)$ \\
\hline Candida albicans & + & $11(21.6) \dagger$ & $7(50.0)$ & $18(27.7)$ \\
\hline Candida krusei & + & $3(5.9)$ & $3(21.4)$ & $6(9.2)$ \\
\hline Candida tropicalis & + & $0(0)$ & $1(7.1)$ & $1(1.5)$ \\
\hline Alcohol sensitivity & Low & $27(52.9)$ & $9(64.3)$ & $36(55.4)$ \\
\hline \multirow[t]{3}{*}{ Smoking status } & Never & $31(60.8)$ & $12(85.7)$ & $43(66.2)$ \\
\hline & Past & $15(29.4)$ & $1(7.1)$ & $16(24.6)$ \\
\hline & Current & $5(9.8)$ & $1(7.1)$ & $6(9.2)$ \\
\hline \multirow[t]{4}{*}{ Drinking frequency (/week)§ } & Never & $18(35.3)$ & $9(64.3)$ & $27(41.5)$ \\
\hline & Light & $26(51.0)$ & $5(35.7)$ & $31(47.7)$ \\
\hline & Moderate & $7(13.7)$ & $0(0)$ & $7(10.8)$ \\
\hline & Heavy & $0(0)$ & $0(0)$ & $0(0)$ \\
\hline $\begin{array}{l}\text { Mean amount of alcohol consumption } \\
\text { (g/day) }\end{array}$ & & $0.7(0.0,1.7) \dagger$ & $0.0(0.0,0.4)$ & $0.6(0.0,1.2)$ \\
\hline \multirow[t]{2}{*}{ Toothbrushing frequency (/day) } & Once & $8(15.7)$ & $2(14.3)$ & $10(15.4)$ \\
\hline & >Twice & $43(84.3)$ & $12(85.7)$ & $55(84.6)$ \\
\hline
\end{tabular}

* Median (25\%, 75\%)

$\dagger p<0.05$, compared to female, chi-square test or Mann-Whitney $U$ test.

$\ddagger$ Number (\%)

$\S$ light = less than 5 days a week; moderate $=5$ or more days a week and less than $360 \mathrm{~mL}$ a day; heavy $=5$ or more days a week and $360 \mathrm{~mL}$ or more a day. 
of the arm for 20 minutes, and was removed in accordance with the manufacturer's instructions. A patch area with erythema after removal was judged to be positive and alcohol sensitivity was considered to be high $(\operatorname{ALDH} 2 * 1 / * 2$ or $* 2 / * 2)$, while in the case of a negative reaction, sensitivity was considered to be low $($ ALDH $2 * 1 / * 1)$.

\section{Questionnaire}

In addition to age, sex and general condition, the questionnaire included the following items: smoking, alcohol consumption and daily frequency of tooth brushing. Because smoking status ${ }^{14}$ can affect acetaldehyde production, we investigated smoking status, which was characterized as "never", "past" and "current"11. Information regarding drinking frequency [never; less than 5 days a week (light); 5 or more days a week, less $360 \mathrm{~mL}$ a day (moderate); 5 or more days a week, $360 \mathrm{~mL}$ or more a day (heavy)], mean amount of alcohol consumption per occasion and type of alcoholic beverage, which included beer, sake, wine, whisky and shochu (distilled alcoholic beverage made from wheat or sweet potatoes) was obtained ${ }^{26}$. We calculated average daily alcohol consumption by multiplying the mean amount of alcohol consumption per occasion by drinking frequency. Alcohol content was estimated to be $20 \mathrm{~g}$ for a bottle of beer $(500 \mathrm{~mL}), 22 \mathrm{~g}$ for a cup of sake $(180 \mathrm{~mL}), 20 \mathrm{~g}$ for a glass of whisky (60 $\mathrm{mL}), 50 \mathrm{~g}$ for a cup of shochu $(180 \mathrm{~mL})$ and $12 \mathrm{~g}$ for a glass of wine $(120 \mathrm{~mL})^{1}$. To assess oral health behavior, participants were asked to state their daily frequency of toothbrushing ${ }^{26}$.

\section{Statistical analysis}

Data analysis was performed using the Statistical Package for Social Science (SPSS version 19) (IBM, Tokyo, Japan). Chi-square test (or Mann-Whitney U test) was performed to compare variables between male and female and to compare acetaldehyde concentration in mouth air between two groups, i.e., male vs. female, Candida species positive vs. negative, low alcohol sensitivity vs. high, nonsmoker vs. smoker, or once a day vs. more than once a day (for toothbrushing frequency) ${ }^{26}$. The association between acetaldehyde concentration and other parameters were analyzed using Spearman correlation coefficient. Wilcoxon signed rank test was used to compare acetaldehyde concentration in mouth air between before and after tongue coating removal and diurnal variation in acetaldehyde concentration (morning, noon and evening). Levels of significance were set at $p<0.05$.

Differences in parameters among the three tongue coating groups (score 0/1, 2 and 3) and the three drinking frequency groups (never, light and moderate) were analyzed by Mann-Whitney $U$ test with Bonferroni correction. Because the number of participants with a tongue coating score of 0 was only 8 , scores of 0 and 1 were combined. The level of significance was set at $p<0.017$.

\section{RESULTS}

Table 1 shows the characteristics of study participants. There were no decayed teeth, severe periodontitis or mucosal lesions. Acetaldehyde concentration in mouth air was $170.7(73.5,306.3)$

Table 2- Differences in acetaldehyde concentration in mouth air

\begin{tabular}{lcc}
\hline \multicolumn{1}{c}{ Variable } & & Acetaldehyde concentration $(\mathrm{ppb})$ \\
\hline Tongue coating status & $0 / 1$ & $87.9(66.9,121.5)$ \\
& 2 & $158.1(74.8,230.5)$ \\
Candida species & 3 & $248.3(172.0,469.4) \dagger$ \\
\hline Alcohol sensitivity & + & $124.2(83.9,242.2)$ \\
& - & $173.6(73.2,341.5)$ \\
Smoking status & Low & $193.7(92.8,347.3)$ \\
& High & $113.2(62.5,248.3)$ \\
Drinking frequency (/week) & Never & $175.1(85.8,342.0)$ \\
& Past/Current & $134.1(69.2,258.9)$ \\
& Never & $192.2(69.4,302.7)$ \\
\hline Toothbrushing frequency (/day) & Light & $175.1(101.7,324.7)$ \\
& Moderate & $124.2(74.2,150.4)$ \\
\hline
\end{tabular}

* Median (25\%, 75\%)

$\dagger p<0.017$, compared to the 0/1 group (tongue coating status), Mann-Whitney $U$ test with Bonferroni correction 
[median (25\%, 75\%)] ppb.

Acetaldehyde concentration in participants with a tongue coating status score of 3 was significantly higher than in those with a score of $0 / 1[248.3$ $(172.0,469.4)$ vs. $87.9(66.9,121.5)](p<0.001)$
(Table 2). Even in participants $(n=31)$ who never smoked and had no Candida species, acetaldehyde concentration in participants with a tongue coating status score of 3 was significantly higher than in those with a score of $0 / 1[97.7(66.0,141.1)$

Table 3- Correlation between acetaldehyde concentration and other parameters

\begin{tabular}{lcc}
\hline \multicolumn{1}{c}{ Variable } & p & p value \\
\hline Age & -0.052 & 0.682 \\
Number of teeth present & 0.102 & 0.42 \\
\hline Mean probing pocket depth $(\mathrm{mm})$ & 0.149 & 0.235 \\
Mean clinical attachment level $(\mathrm{mm})$ & 0.159 & 0.206 \\
\hline Bleeding on probing $(\%)$ & -0.049 & 0.698 \\
Plaque control record $(\%)$ & 0.132 & 0.296 \\
\hline Mean amount of alcohol consumption (g/day) & 0.056 & 0.661 \\
\hline
\end{tabular}

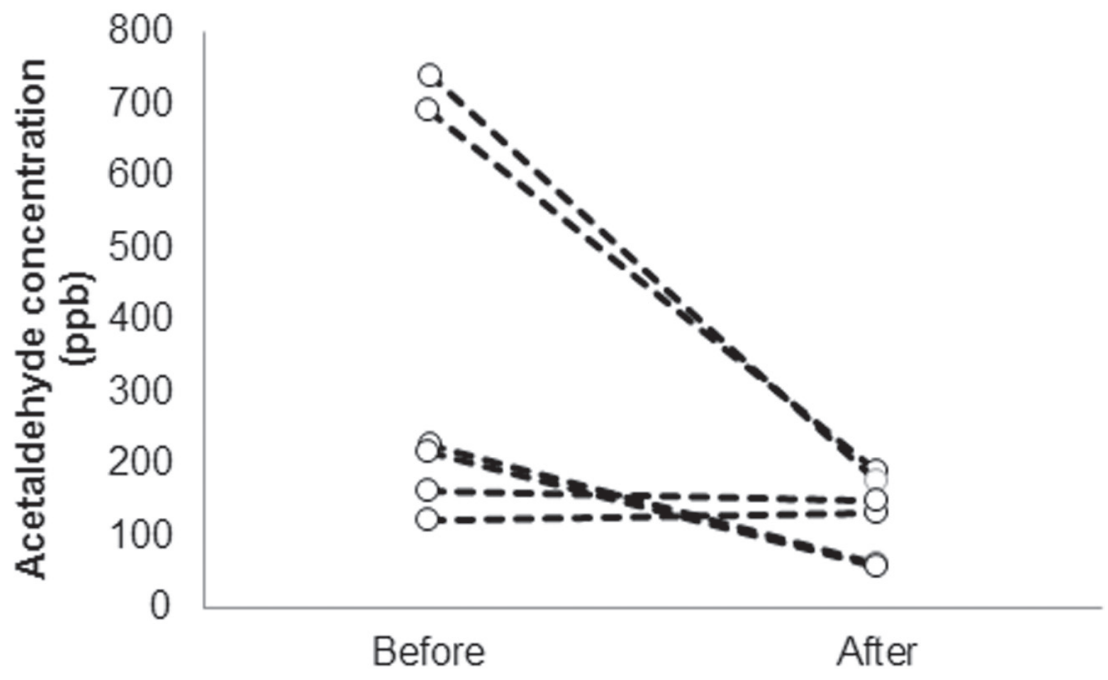

Figure 2- Acetaldehyde concentration in mouth air before and after tongue coating removal. Acetaldehyde concentration decreased significantly after tongue coating removal (Wilcoxon signed rank test, $p<0.05, n=6$ )

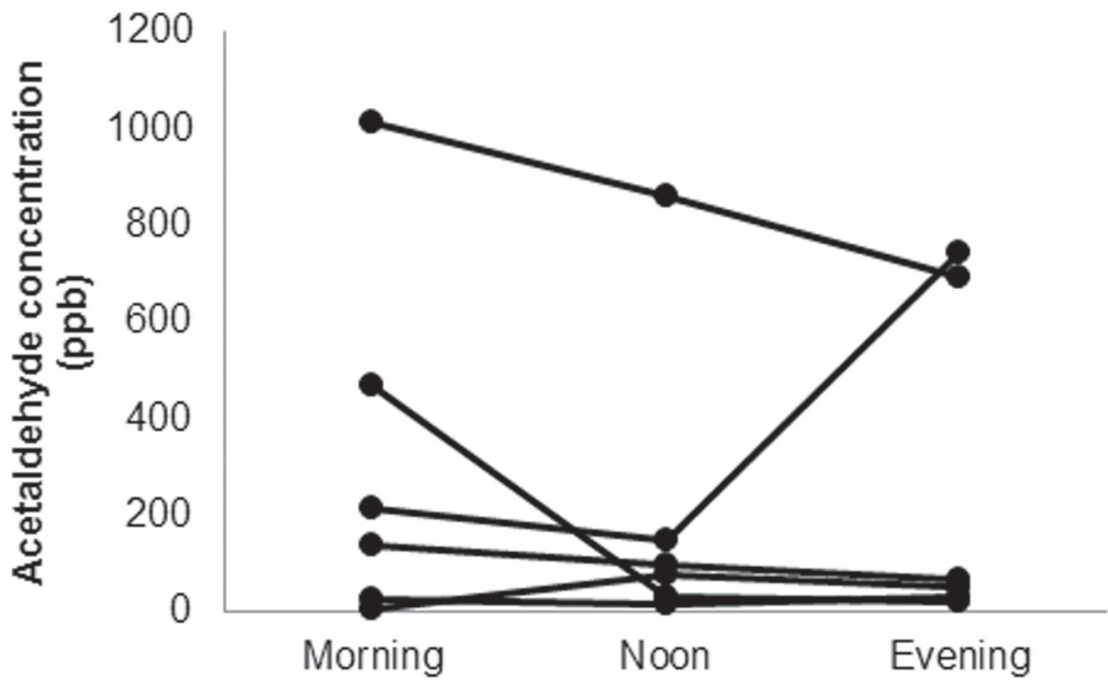

Figure 3- Diurnal variation in acetaldehyde concentration (morning, noon and evening). Acetaldehyde concentration did not present significant diurnal variations (Wilcoxon signed rank test, $p>0.05, n=6$ ) 
vs. $315.8(209.3,579.4)](p<0.001)$. There were no significant differences in acetaldehyde concentration between other parameters, including alcohol sensitivity and drinking habits (Table 2). No parameters were associated with acetaldehyde concentration (Table 3).

Acetaldehyde concentration decreased significantly after tongue coating removal [222.0 (176.2, 575.5) vs. $141.9(80.5,170.1)$ ] (Figure 2) $(p<0.05)$. Acetaldehyde concentration did not exhibit significant diurnal variations $[177.5$ (53.8, $406.6)$ in the morning, $86.5(42.8,136.0)$ at noon and $61.7(37.9,536.0)$ in the evening] (Figure 3 ) $(p>0.05)$.

\section{DISCUSSION}

Although one study has reported data on physiological concentrations of acetaldehyde in human breath (0.4-1.6 ppb) ${ }^{5}$, the relationship between its concentration and oral condition, which may affect local production of acetaldehyde, is uncertain. In this study, acetaldehyde concentration in mouth air was significantly related to tongue coating status. Furthermore, removing tongue coating significantly decreased acetaldehyde concentration. These results suggest that one of the sources of acetaldehyde in mouth air is tongue coating.

Acetaldehyde concentration in participants with a tongue coating status score of 3 was significantly higher than in those a score of $0 / 1$. Acetaldehyde is produced in the epithelium by mucosal ALDH ${ }^{2}$, and higher levels of acetaldehyde are derived from microbial oxidation of ethanol by oral microflora9, 10,12,17,23. In this study, acetaldehyde concentration was associated with tongue coating scores, but not plaque scores. Tongue coating serves as a reservoir for oral microflora ${ }^{24}$, thus, oral microflora on the tongue may be the main source of local production of acetaldehyde, rather than endogenous metabolic activity. However, we did not investigate the bacterial species on the tongue, which is a limitation of this study.

It may be important for oral health to reduce acetaldehyde concentrations in mouth air. The causes of halitosis are largely located in the mouth and can be attributed to tongue coating and periodontal disease ${ }^{29}$. Removing tongue coating improves oral malodor, which reduces acetaldehyde concentration and prevents mutations in DNA. However, further studies are required to clarify the details.

Candida species possess ALDH and are a source of acetaldehyde generation ${ }^{15}$. The production of carcinogenic acetaldehyde by Candida species has been suggested to contribute to oral carcinogenesis ${ }^{7,13}$. However, in this study, acetaldehyde concentration was not associated with the presence of Candida species. The discrepancy between the results of these studies and the present study may be due to differences in the procedure for detection of acetaldehyde concentration and in experimental conditions; we directly detected the physiological concentration in mouth air, while other studies incubated Candida species with ethanol, and acetaldehyde production was then measured ${ }^{7,13}$. As Neisseria ${ }^{20}$ and Streptococci ${ }^{13}$ strains can also be a regional source of acetaldehyde, further studies are required to investigate the main acetaldehydeproducing bacteria.

Alcohol sensitivity ${ }^{16}$, amount of alcohol consumption ${ }^{3}$ and smoking status ${ }^{14}$ can affect acetaldehyde production. On the other hand, ALDH2 genotype had no effect on salivary acetaldehyde before and after ethanol exposure ${ }^{8}$. Smoking status did not result in differences in the production of acetaldehyde in saliva ${ }^{6}$. In this study, acetaldehyde concentration was not associated with alcohol sensitivity, drinking habits or smoking status. The discrepancies between our study and previous studies are unclear. However, alcohol sensitivity, amount of alcohol consumption and smoking status may not affect acetaldehyde production in mouth air, as participants refrained from smoking and from drinking alcohol for $12 \mathrm{~h}$ prior to measurements, and the main source of acetaldehyde is oral microflora.

Acetaldehyde concentration [Median (25\%, $75 \%)]$ in mouth air was $170.7(73.5,306.3) \mathrm{ppb}$ in this study. The values were higher than in a previous study, which reported that the physiological concentration of acetaldehyde in human breath was 0.4-1.6 ppb using gas chromatography ${ }^{5}$. The difference may be due to experimental conditions. We measured the concentration in the morning under limited conditions for easier detection. Further investigations are necessary to monitor slight physiological changes or diurnal variations for low acetaldehyde levels (ppb order) in mouth air using a more sensitive portable monitor.

This study has other limitations. First, all subjects were recruited at Okayama University Hospital, and the number of subjects was small, which may limit the ability to extrapolate these findings to the general population. Second, although acetaldehyde concentration significantly decreased after tongue coating removal, the number of participants was small. Additional large-scale studies will thus provide information beyond the findings presented here.

\section{CONCLUSION}

This study revealed that acetaldehyde concentration in mouth air is associated with tongue coating, which may be a source of local production 
by oral microflora.

\section{ACKNOWLEDGMENTS}

This work was supported by Grants-in-Aid for Scientific Research (24593154) from the Ministry of Education, Culture, Sports, Science and Technology, Tokyo, Japan. We are also grateful to Mr. Gohei Oyama and Mr. Shuhei Uno (Okayama University, Okayama, Japan) for helping with data entry.

\section{REFERENCES}

1- Asakage T, Yokoyama A, Haneda T, Yamazaki M, Muto M, Yokoyama T, et al. Genetic polymorphisms of alcohol and aldehyde dehydrogenases, and drinking, smoking and diet in Japanese men with oral and pharyngeal squamous cell carcinoma. Carcinogenesis. 2007;28:865-74.

2- Chiang CP, Jao SW, Lee SP, Chen PC, Chung CC, Lee SL, et al. Expression pattern, ethanol-metabolizing activities, and cellular localization of alcohol and aldehyde dehydrogenases in human large bowel: association of the functional polymorphisms of $A D H$ and ALDH genes with hemorrhoids and colorectal cancer. Alcohol. 2012;46:37-49

3- Dagli RJ, Kulkarni S, Duraiswamy P, Dagli NR, Khara NV, Khara $\mathrm{BN}$. Is alcohol an independent risk factor for oro-pharyngeal and pulmonary carcinogenesis - an acetaldehyde concentrations based double blinded randomized control trial. J Int Oral Health. 2013;5:62-7.

4- Dong YJ, Peng TK, Yin SJ. Expression and activities of class IV alcohol dehydrogenase and class III aldehyde dehydrogenase in human mouth. Alcohol. 1996;13:257-62.

5- Eriksson CJ. Measurement of acetaldehyde: what levels occur naturally and in response to alcohol? Novartis Found Symp. 2007;285:247-55.

6- Ernstgård L. Influence of gender on the metabolism of alcohols in human saliva in vitro. Arch Oral Biol. 2009;54:737-42.

7- Gainza-Cirauqui ML, Nieminen MT, Frazer LN, Aguirre-Urizar JM, Moragues MD, Rautemaa R. Production of carcinogenic acetaldehyde by Candida albicans from patients with potentially malignant oral mucosal disorders. J Oral Pathol Med. 2013;42:2439.

8- Helminen A, Väkeväinen S, Salaspuro M. ALDH2 genotype has no effect on salivary acetaldehyde without the presence of ethanol in the systemic circulation. PLoS One. 2013;8:e74418.

9- Homann N, Jousimies-Somer $H$, Jokelainen $K$, Heine $R$, Salaspuro M. High acetaldehyde levels in saliva after ethanol consumption: methodological aspects and pathogenetic implications. Carcinogenesis. 1997; 18:1739-43.

10- Homann N, Tillonen J, Meurman JH, Rintamäki H, Lindqvist C, Rautio $\mathrm{M}$, et al. Increased salivary acetaldehyde levels in heavy drinkers and smokers: a microbiological approach to oral cavity cancer. Carcinogenesis. 2000;21:663-8.

11- Kim MJ, Son KH, Park HY, Choi DJ, Yoon CH, Lee HY, et al. Association between shift work and obesity among female nurses: Korean Nurses' Survey. BMC Public Health. 2013;13:1204.

12- Kurkivuori J, Salaspuro V, Kaihovaara P, Kari K, Rautemaa R, Grönroos L, et al. Acetaldehyde production from ethanol by oral streptococci. Oral Oncol. 2007;43:181-6.
13- Marttila E, Bowyer P, Sanglard D, Uittamo J, Kaihovaara P, Salaspuro M, et al. Fermentative 2-carbon metabolism produces carcinogenic levels of acetaldehyde in Candida albicans. Mol Oral Microbiol. 2013;28:281-91.

14- Marttila E, Uittamo J, Rusanen P, Lindqvist C, Salaspuro M, Rautemaa R. Acetaldehyde production and microbial colonization in oral squamous cell carcinoma and oral lichenoid disease. Oral Surg Oral Med Oral Pathol Oral Radiol. 2013;116:61-8.

15- Meurman JH, Uittamo J. Oral micro-organisms in the etiology of cancer. Acta Odontol Scand. 2008;66:321-6.

16- Mitsubayashi K, Matsunaga H, Nishio G, Toda S, Nakanishi Y. Bioelectronic sniffers for ethanol and acetaldehyde in breath air after drinking. Biosens Bioelectron. 2005;20:1573-9.

17- Miyakawa H, Baraona E, Chang JC, Lesser MD, Lieber CS. Oxidation of ethanol to acetaldehyde by bronchopulmonary washings: role of bacteria. Alcohol Clin Exp Res. 1986;10:517-20. 18- Miyazaki H, Sakao S, Katoh Y, Takehara T. Correlation between volatile sulphur compounds and certain oral health measurements in the general population. J Periodontol. 1995;66:679-84.

19- Murata T, Rahardjo A, Fujiyama Y, Yamaga T, Hanada M, Yaegaki $K$, et al. Development of a compact and simple gas chromatography for oral malodor measurement. J Periodontol. 2006;77:1142-7.

20- Muto M, Hitomi $Y$, Ohtsu A, Shimada H, Kashiwase $Y$, Sasak $\mathrm{H}$, et al. Acetaldehyde production by non-pathogenic Neisseria in human oral microflora: implications for carcinogenesis in upper aerodigestive tract. Int J Cancer. 2000;88:342-50.

21- Nadeem SG, Hakim ST, Kazmi SU. Use of CHROMagar Candida for the presumptive identification of Candida species directly from clinical specimens in resource-limited settings. Libyan J Med. 2010;5. doi: 10.3402/ljm.v5i0.2144

22- O'Leary TJ, Drake RB, Naylor JE. The plaque control record. J Periodontol. 1972;43:38.

23- Pikkarainen PH, Baraona E, Jauhonen P, Seitz HK, Lieber CS. Contribution of oropharynx microflora and of lung microsomes to acetaldehyde in expired air after alcohol ingestion. J Lab Clin Med. 1981;97:631-6.

24- Roldán S, Herrera D, Sanz M. Biofilms and the tongue: therapeutical approaches for the control of halitosis. Clin Oral Investig. 2003;7:189-97.

25- Salaspuro MP. Acetaldehyde, microbes, and cancer of the digestive tract. Crit Rev Clin Lab Sci. 2003;40:183-208.

26- Sato F, Oze I, Kawakita D, Yamamoto N, Ito H, Hosono S, et al. Inverse association between toothbrushing and upper aerodigestive tract cancer risk in a Japanese population. Head Neck. 2011;33:1628-37.

27- Stavenow L, Jerntorp $\mathrm{P}$, Ohlin $\mathrm{H}$. Effects of ethanol and acetaldehyde on cultured rabbit aortic myocytes and human platelets in vitro. Eur J Clin Invest. 1984;14:242-6.

28- Tsutaya S, Shoji M, Saito Y, Kitaya H, Nakata S, Takamatsu H, et al. Analysis of aldehyde dehydrogenase 2 gene polymorphism and ethanol patch test as a screening method for alcohol sensitivity. Tohoku J Exp Med. 1999;187:305-10.

29- Van den Broek AM, Feenstra L, de Baat C. A review of the current literature on management of halitosis. Oral Dis. 2008; 14:30-9. 